\title{
REAL-TIME RENDERING WITH HETEROGENEOUS GPUS
}

\author{
Xiao Lei and Vetria L. Byrd \\ Purdue University, Department of Computer Graphics Technology \\ 401 North Grant Street, West Lafayette, IN 47907, United States
}

\begin{abstract}
A large portion of recent personal computers are equipped with both integrated and discrete GPUs. However, most of the time these two GPUs are not utilized concurrently in the same application. This paper provides an exploration into utilizing integrated plus discrete GPUs to improve real-time rendering performance with Vulkan API. Specifically, our approach includes offloading asynchronous computation workload from the discrete GPU to the integrated GPU, and multi-threading and render graphs are adopted to saturate GPUs utilization. Through benchmarking, we validate that our approach can speed up the overall performance by up to $4 \%$ with the utilization of combinations like Intel UHD 630 plus NVIDIA RTX 2070. Our results reveal that with tools like Vulkan, such heterogeneous-GPU system is exploitable.
\end{abstract}

\section{KEYWORDS}

Heterogeneous-GPU, Vulkan API, Rendering Performance

\section{INTRODUCTION}

With previous API like OpenGL, an application running on a computer equipped with both integrated and discrete GPUs cannot make use of both GPUs without context switching. This makes either the integrated or discrete GPU remain idle while the other GPU is loaded. This scenario suggests that there could be potential benefits if all the available GPUs can be enabled.

The introduction of low-level command and resource control brought by Vulkan makes it possible to manage resource and task scheduling across vendor-independent graphics hardware in application-level. This explicit control inspired the idea of utilizing modern personal computers' heterogeneous GPUs (integrated plus discrete) feature to obtain performance improvement.

A similar approach for exploiting parallelism offered by integrated GPU (Peek, et al., 2014) has been applied to head-mounted displays, in which the integrated GPU is utilized to do image warping. A former multi-adapter experiment (Yeung, 2015) also claimed a noticeable framerate improvement with the discrete plus integrated GPUs combination. In recent GDC 2020, Intel presented their idea (Hux, 2020) of utilizing integrated GPU to speed up the n-Body particle rendering. Despite these advances, the literature shows there is very little overlap with implementing heterogeneous GPUs system with Vulkan and evaluating the outcome.

This paper aims to identify, design, and evaluate the methods that could allow the integrated GPU to assist real-time rendering through offloading workloads from the discrete GPU with Vulkan implementation. Towards achieving these aims, we implemented and benchmarked a real-time rendering program based on Vulkan and $\mathrm{C}++$. The program resembles a pure graphics engine that supports $3 \mathrm{D}$ rendering with several shading techniques and could offload certain workloads from the discrete GPU to the integrated GPU. 


\section{METHODS}

\subsection{Offloading Workload from Discrete GPU}

Through the physical device querying and logical feature wrapping interfaces provided by Vulkan, the integrated GPU (iGPU) and the discrete GPU (dGPU) can be encapsulated as two logical devices.

Since the resources created on different logical devices are not interchangeable, the heterogeneous GPUs renderer manages two sets of resources: one set used by the dGPU and the other set used by the iGPU. Data from one GPU is transferred to the other GPU through explicit buffer copying. This schema is illustrated in Figure 1. Under such schema, swapchain presentation is handled by the dGPU, and there is no read back from video memory to system memory as all data transfers are unidirectional.

In each frame, certain application data is copied into iGPU resources to perform asynchronous update, while the original values from the previous frame are used for output image rendering in the dGPU. The input data is read and written as an image in the iGPU.

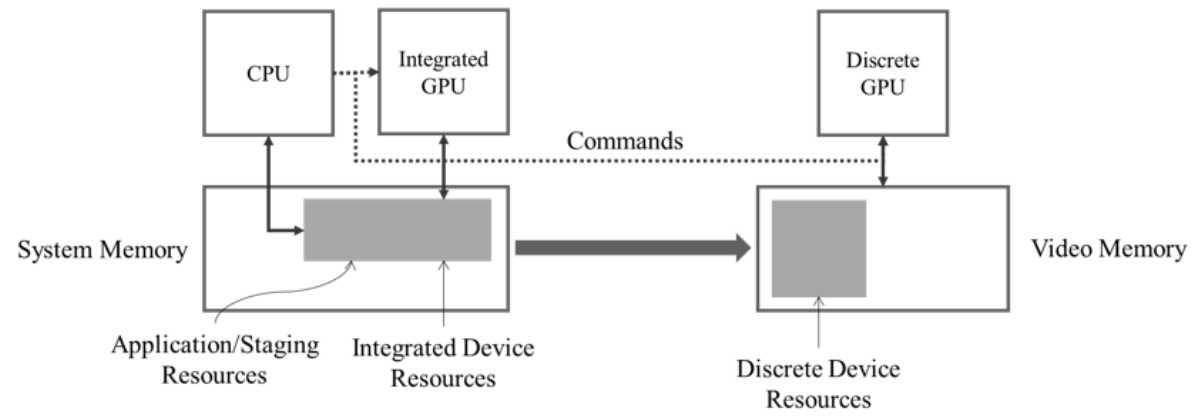

Figure 1. Integrated GPU works on asynchronous computation

\subsection{Saturating the GPUs}

One of the valuable features that Vulkan provides is improved compatibility with multi-threading. To better utilize this multi-threading capability, render graph (Persson, 2017) is adopted in our approach.

A render graph is an acyclic graph that describes the render pass behaviors and organizes their dependencies. The basic unit of a render graph is pass node. Each pass node consists of 5 components: node properties, render context, input resources, output resources, and render pass functions. The two GPUs (iGPU and dGPU) each own an exclusive render graph specifically for task description.

Multi-threading can be implemented on top of the render graph as illustrated in Figure 2. During one frame, each command-recording thread takes one pass node from the render graph by priority sequence and executes the pass functions. All the command recording threads hold individual command buffer pools for command buffer allocation.

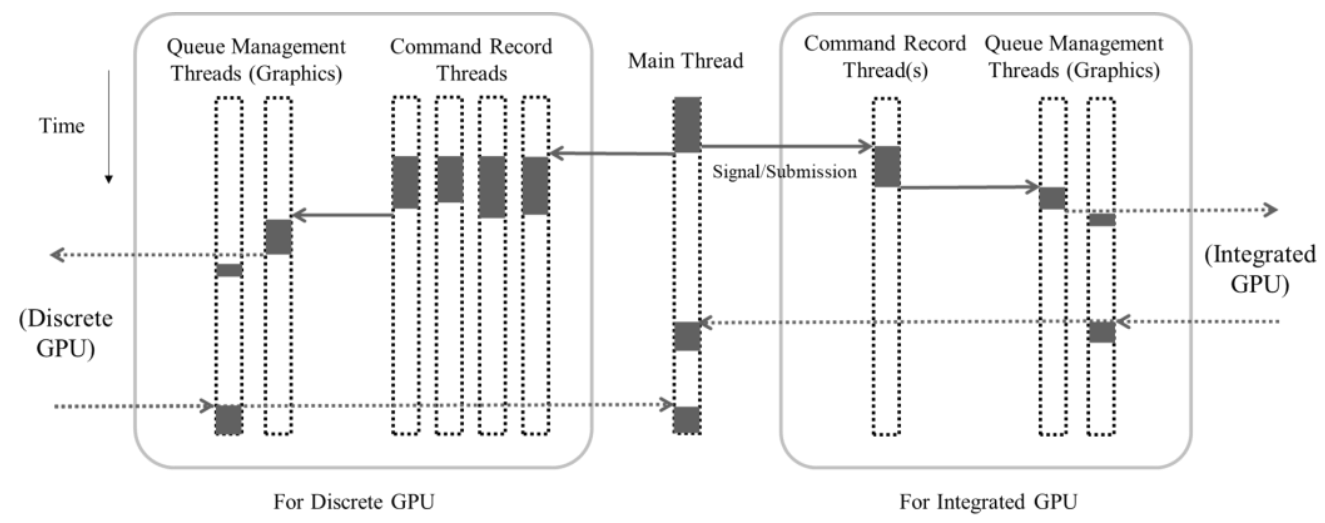

Figure 2. Multi-threading design for heterogeneous GPUs 
When recording with multiple threads, the finished command buffers may arrive in any sequence. The renderer running on the main thread or cycle management thread is therefore responsible for checking and holding each arrived command buffer and ensuring they are submitted in the correct sequence.

The main thread is in synchronization with the dGPU since the dGPU directly controls when a frame is finally presented. Since in our settings the iGPU is expected to complete its job inside the same frame, the two GPUs work under the same task execution cycle in parallel. Timeline semaphore is used as the main synchronization primitive between CPU and GPU.

The complete process can be outlined as follows:

\section{Begin $\downarrow$}

Query physical devices and setup logical devices

\section{Initialize $\downarrow$}

Generate render graphs and allocate corresponding resources

\section{Frame Loop $\bigcirc$}

Copy asynchronous task data to iGPU's buffer

Generate and submit command buffers to iGPU and dGPU (parallel)

Copy results back from iGPU's buffer (parallel)

Recycle frame resources (parallel)

Present final image on dGPU

\section{RESULTS AND DISCUSSION}

A scene with 512 objects was used during the test, as shown in Figure 3. Each object is a textured amago fish mesh with $6.3 \mathrm{~K}$ triangles. In each frame, the positions of all the objects are put into asynchronous update while the original values are used for rendering in the current frame. The position data is read and written as a $32 \times 16$ image in the iGPU. Two personal computers with different hardware combinations were used as testing devices. Their detailed specifications are given in Table 1.

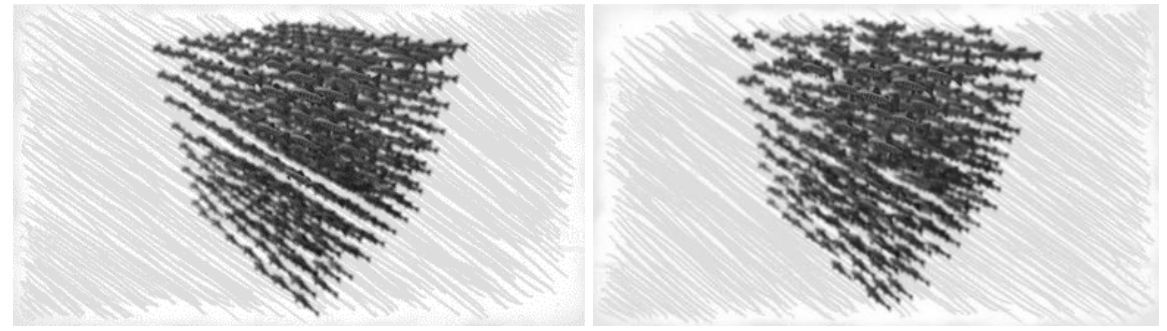

Figure 3. Screenshot: the test scenario with 512 objects. Background strokes are part of the post-processing step and are not related to asynchronous computation

With default test workload, the average (discrete) GPU frame time on device A is $6.6 \mathrm{~ms}$ and this value is $19 \mathrm{~ms}$ on device B. The fraction taken up by the asynchronous computation step on device A and B are $0.8 \mathrm{~ms}$ and $2.3 \mathrm{~ms}$, respectively. When applying Amdahl's law (Hill \& Marty, 2008) in the theoretical estimation, the fraction of asynchronous computation can be treated as completely taken out from the sequential workflow. The speedup is also upper bounded by hardware capabilities as listed in individual GPU theoretical performance in Table 1. Thus, the final theoretical speedup upper bound would be 1.080 for device A $(0.441 / 5.46+1.0)$ and 1.137 for device B $(1.0 /(1.0-(2.3 / 19.0)+0.0))$. 
Table 1. Specifications of the testing devices

\begin{tabular}{|c|c|c|}
\hline & Device A & Device B \\
\hline CPU & Intel Core i7 9750H & Intel Core i5 7300HQ \\
\hline Discrete GPU & NVIDIA GeForce RTX 2070 Max-Q & NVIDIA GeForce GTX 1050 \\
\hline Performance (discrete) & 5.460 TFLOPS & 1.911 TFLOPS \\
\hline Integrated GPU & Intel UHD Graphics 630 & Intel HD Graphics 630 \\
\hline Performance (integrated) & 441.6 GFLOPS & 384.0 GFLOPS \\
\hline System Memory & DDR4 2666 16GB 64-bit $\times 2$ & DDR4 2133 8GB 64-bit \\
\hline Vulkan Runtime & 1.2 .131 .2 & 1.2 .131 .2 \\
\hline
\end{tabular}

The simulated workload consists of a series of trigonometric and pseudo-random computations. The default test load contains 5000 computation loops, and the extra test case (4×load) increased this loop count to 20000 . Note that the load increase is not necessarily proportional to the actual time to complete the asynchronous task.

The results measured on both devices are presented in Table 2. The major additional overhead comes from issuing commands for the iGPU, which would delay the command recording for the dGPU, as illustrated in Figure 5. In the meantime, as depicted in Figure 4, this overhead can get covered by the workload fraction taken out from the dGPU.

Table 2. Measured average frame time in milliseconds (lower is better)

\begin{tabular}{|c|c|c|c|c|}
\hline & Device A & Device A (4×load) & Device B & Device B (4×load) \\
\hline Discrete GPU & $6.913 \mathrm{~ms}$ & $7.231 \mathrm{~ms}$ & $20.063 \mathrm{~ms}$ & $20.134 \mathrm{~ms}$ \\
\hline Heterogeneous GPUs & $6.639 \mathrm{~ms}$ & $9.329 \mathrm{~ms}$ & $19.708 \mathrm{~ms}$ & $19.498 \mathrm{~ms}$ \\
\hline
\end{tabular}

In three of the four test cases, a maximum of $4.1 \%$ frame time improvement is observed. Although in the second case there is a performance downgrade, it can be expected with the given test conditions. The default workload would take the iGPU on device A about $5 \mathrm{~ms}$ to complete, which is within the time required by the dGPU to complete a full frame. But as the computational load increased, the iGPU became the bottleneck on device A, resulting in the performance decrease. In the meantime, the iGPU on device B only has slightly lower performance than the iGPU on device A, thus the time required by the iGPU on device B to complete the same computational task can be expected to be well within $19 \mathrm{~ms}$ when the computational load increased.

Such observation suggests that when offloading asynchronous computation workload to the iGPU, the relative performance of the iGPU and application target frame rate should be carefully balanced.

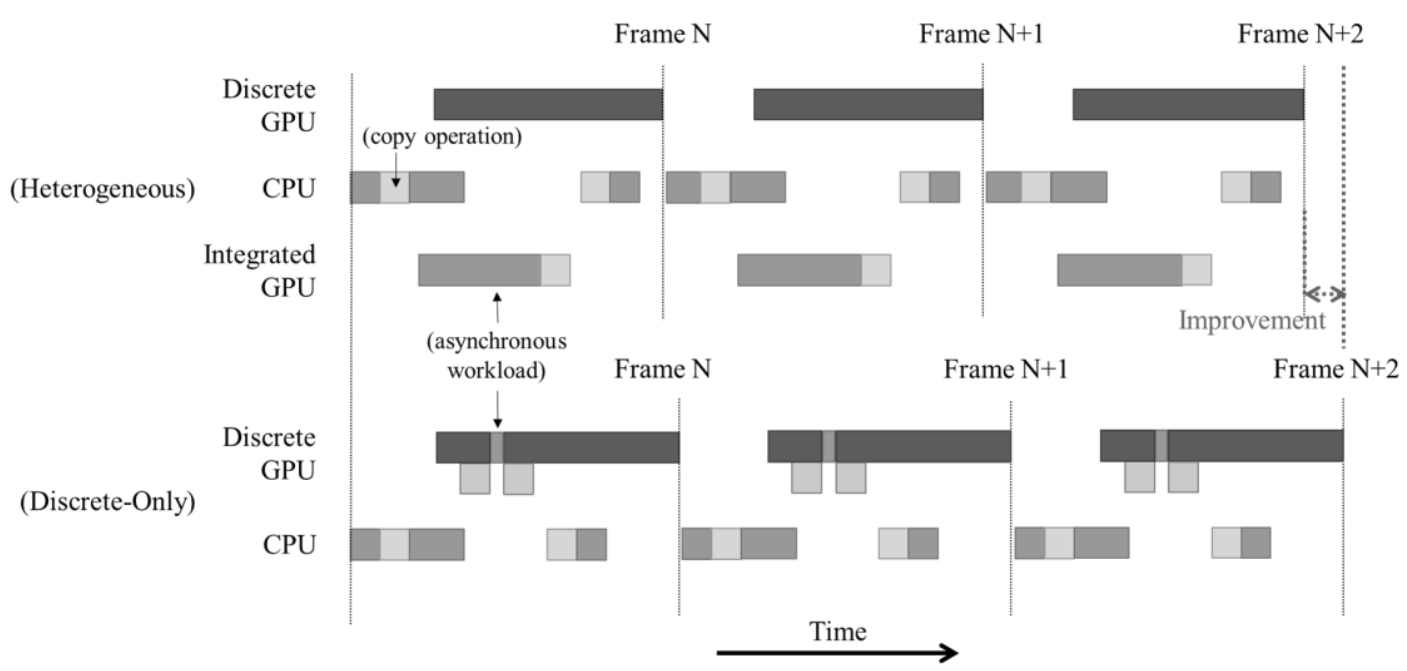

Figure 4. Source of frame time improvement. This is an illustration, not accurate time mapping 


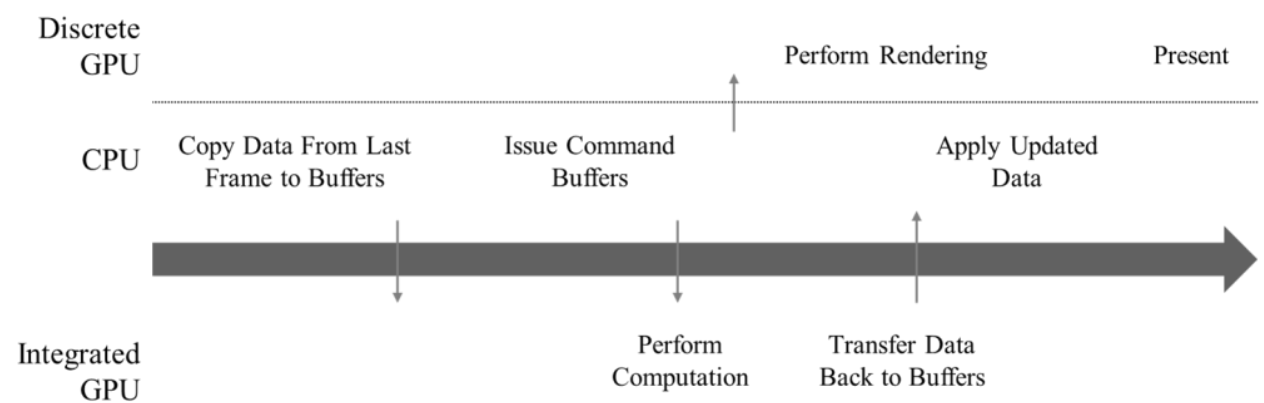

Figure 5. Timeline of a frame with heterogeneous GPUs

Aside from asynchronous computation workload, we have also experimented offloading post-processing workload that includes copying 3 full-screen images from dGPU to iGPU. However, due to the limit of PCI-e transfer bandwidth, the average data transfer overhead $(5 \mathrm{~ms}$ in total) exceeds the workload fraction that can be offloaded from the dGPU. The best outcome we observed is an average of $8.9 \%$ framerate downgrade comparing with single dGPU setup (39.572ms/frame vs. $36.324 \mathrm{~ms} /$ frame) on device A.

\section{CONCLUSION}

This paper validates that offloading asynchronous computation workload from the dGPU to the iGPU, with Vulkan implementation, can provide up to $4 \%$ performance improvement. Tested combinations include Intel UHD 630 plus NVIDIA RTX 2070, and Intel HD 630 plus NVIDIA GTX 1050.

The necessary conditions include:

1. The data transfer overhead between the iGPU and the dGPU must be less than the workload fraction taken out from the dGPU.

2. The iGPU must be able to complete the offloaded task within the time required by the dGPU to complete a full frame unless the task is not frame-aligned.

This work contributes to the body of knowledge on real-time rendering with heterogeneous GPUs. The approach presented in this work is applicable in graphics applications with asynchronous procedures involved. Examples include character flock behavior update, particle physics simulation with low or fixed refresh rate, and resource pre-processing. Our approach can be further improved through analyzing the performance stability when other GPU-demanding program is present in the system and identifying the sweet spot in the size of data transferred for the offloaded workload.

\section{REFERENCES}

Hill, M. D., \& Marty, M. R. (2008). Amdahl's Law in the Multicore Era. Computer, 41(7), 33-38.

Hux, A. (2020, March 17). Multi-Adapter with Integrated and Discrete GPUs. Retrieved from https://devmesh.intel.com/projects/multi-adapter-particles

Peek, E., Wünsche, B., \& Lutteroth, C. (2014). Using Integrated GPUs to Perform Image Warping for HMDs. Proceedings of the 29th International Conference on Image and Vision Computing New Zealand, 172-177.

Persson, T. (2017, August 28). High-Level Rendering Using Render Graphs. Retrieved from Our Machinery: https://ourmachinery.com/post/high-level-rendering-using-render-graphs/

Yeung, A. (2015, April 30). DirectX 12 Multiadapter: Lighting up dormant silicon and making it work for you. Retrieved from DirectX Developer Blog: https://devblogs.microsoft.com/directx/directx-12-multiadapter-lighting-up-dormantsilicon-and-making-it-work-for-you/ 